\title{
Cracking of GaN Based III-Nitride Heterostructures Grown by MOVPE on (0001)-6H-SiC
}

\author{
A. Hasenkopf, ${ }^{\mathrm{a}, \mathrm{b}}$ F. Scholz, ${ }^{\mathrm{b}}$ F. Phillipp ${ }^{\mathrm{a}}$ \\ ${ }^{a}$ Max-Planck-Institut für Metallforschung, Heisenbergstr. 3, 70569 Stuttgart, Germany \\ b 4. Physikalisches Institut, Universität Stuttgart, Pfaffenwaldring 57, 70550 Stuttgart, Germany
}

During the last years, GaN based III-nitrides have been intensively studied due to their potential applications in both short-wavelength range optoelectronics and high speed, high power electronics. Although notable developments have already been achieved [1] there are still a lot of difficulties to be overcome. One of the problems to be controlled is strain which arises because of a large lattice mismatch between $\mathrm{GaN}$ films and the commonly used substrates (sapphire or SiC), additionally strengthened by largely differing thermal expansion coefficients. As a result, nitride heterostructures commonly contain dislocation densities up to $10^{9} \mathrm{~cm}^{-2}$ and more and can sometimes even suffer cracking $[2,3]$. As cracks strongly limit the design of laser diodes and therefore should be avoided it is necessary to understand cracking mechanisms.

Complex layered GaN/InGaN/AlGaN laser structures grown by MOVPE on (0001)-6H-SiC substrates in some cases already showed cracking at a total thickness of less than $2 \mu \mathrm{m}$. Transmission electron microscopy (TEM) investigations were performed on these laser structures in order to reveal details of crack propagation and to specify conditions under which cracks preferably occur. TEM cross-sectional as well as plan-view specimens were prepared in a conventional way. The specimens were examined in a Philips CM20 transmission electron microscope operating at $200 \mathrm{kV}$ using bright field two-beam diffraction conditions. Before starting the TEM preparation process the samples' surfaces were firstly examined by means of an optical microscope and already at this stage showed clear indication of cracking (Fig. 1a). The geometrical arrangement of dark lines at an average distance of $20 \mu \mathrm{m}$ obviously reflects the hexagonal crystal structure of $\mathrm{GaN}$ and is associated to cracking along the (10-10) planes. This assumption is verified by TEM plan-view investigations (Fig. 1b). Interference fringes visible in Fig. 1a even suggest that the multilayer partially or completely flakes off the substrate. TEM cross-sectional samples indeed show large cracks that propagate nearly parallel to the substrate/multilayer interface and cause large parts of the multilayer to flake off (Fig. 2). They originate from another type of cracks running along the (10-10) planes and mostly, but not exclusively, form in the lower parts of the heterostructure. Interestingly it can be seen that they do not run along heterointerfaces (Fig. 3) where strain due to lattice mismatch should be dominating. Typical dislocation arrangements show dislocations passing the cracks almost without any interruption (Fig. 3) and therefore prove that cracking occurs mainly after the growth process. Therefore different thermal expansion is suggested to be the main reason for cracking of GaN multiple heterostructures.

\section{References}

[1] S. Nakamura, G. Fasol, The Blue Laser Diode - GaN Based Light Emitters and Lasers, Springer, Berlin, 1997

[2] S. Yamaguchi et al., Appl. Phys. Lett. Vol. 79 No. 19 (2001) 3062

[3] J. Yamamoto et al., J. Cryst. Growth Vol. 189/190 (1998) 193 
[5] This work has been supported by the Deutsche Forschungsgemeinschaft (DFG) in the Graduate Program 'Interfaces in Crystalline Materials' (GRK 285).
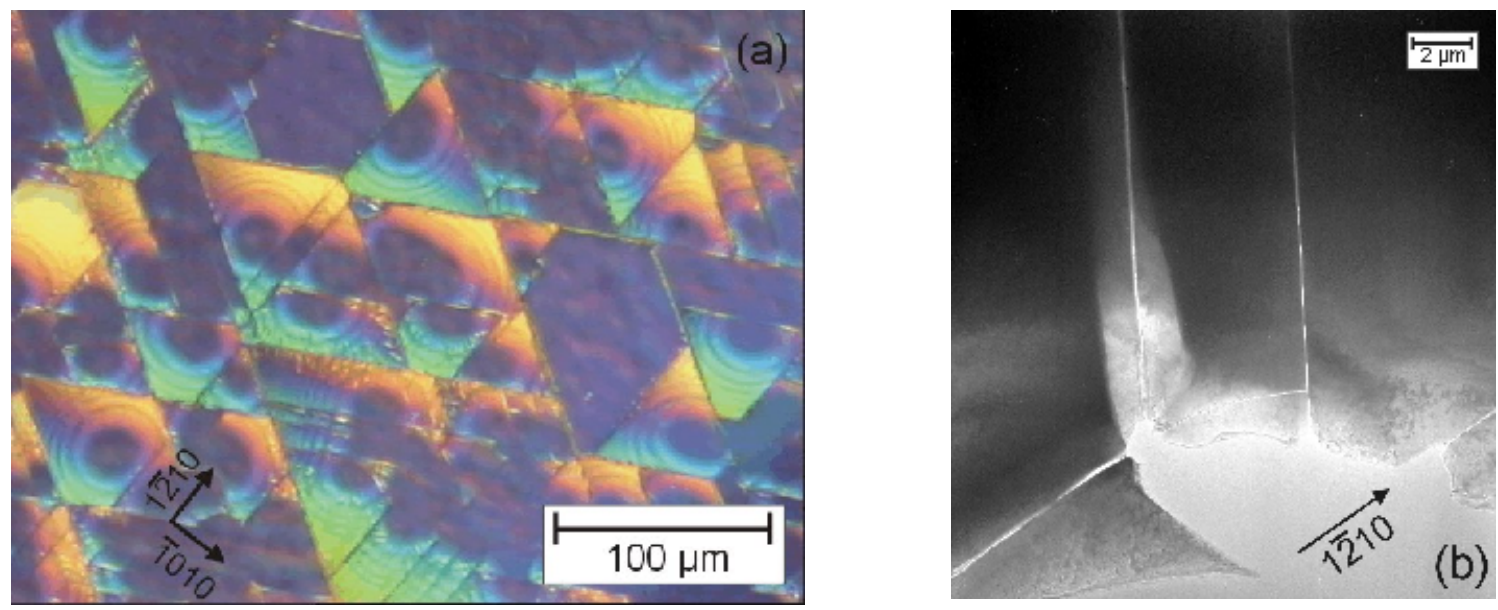

FIG. 1. Optical micrograph of the surface of a GaN multilayer laser structure showing cracks and interference fringes (a) and TEM plan-view micrograph of the same sample confirming the cracks running along the crystallographic directions (b).
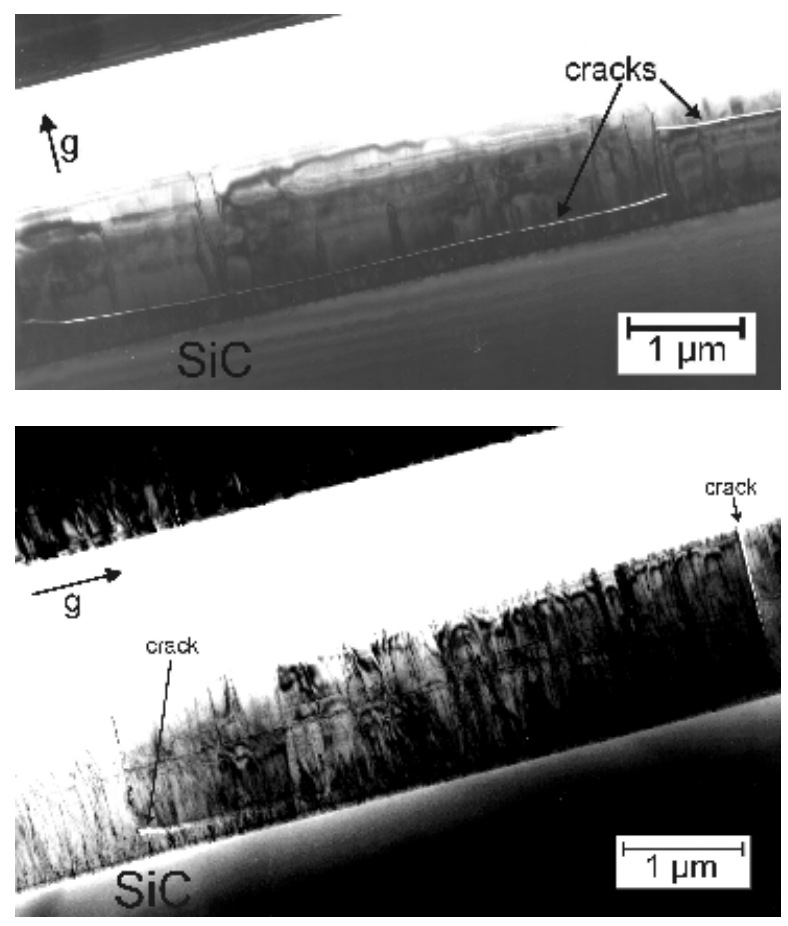

FIG. 2. TEM cross section micrographs of two heterostructures showing both cracks running almost parallel to the (0001) plane and along the (0001) direction.

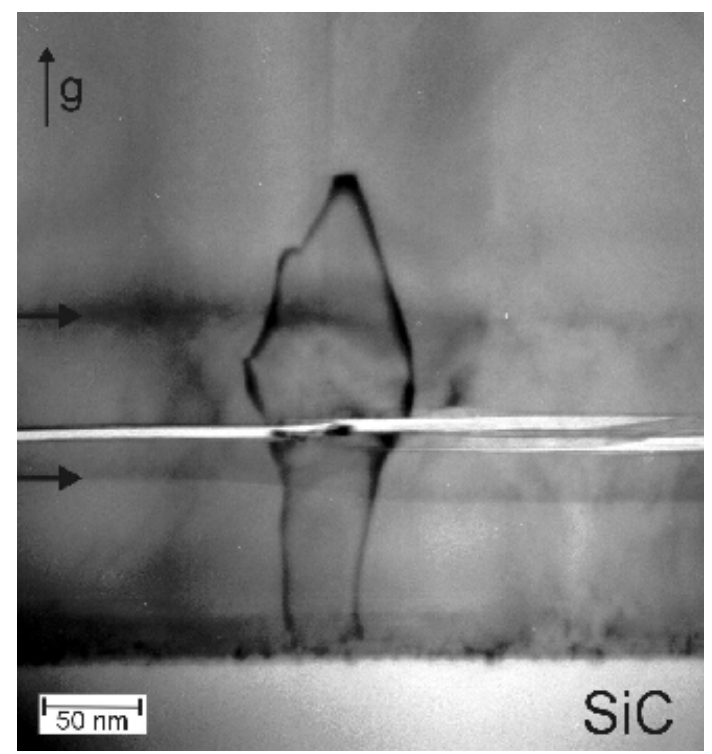

FIG. 3. Severe crack in a laser structure parallel to the (0001) plane. It clearly does not run along a heterointerface two of which are visible (marked by arrows). A dislocation is steadily passing the crack. 